\title{
Effect of substrate temperature on the microstructure of thin niobium films
}

\author{
C. Sürgers, C. Strunk and H. v. Löhneysen \\ Physikalisches Institut, Universität Karlsruhe, Engesser Str. 7, D-76128 Karlsruhe (Germany)
}

(Received July 14, 1993; accepted September 8, 1993)

\begin{abstract}
Niobium films with constant thickness have been deposited on sapphire (1120) by electron-beam evaporation at different substrate temperatures $\left(150^{\circ} \mathrm{C} \leqslant T_{\mathrm{S}} \leqslant 750^{\circ} \mathrm{C}\right)$. The samples were characterized by $\mathrm{X}$-ray diffraction and resistivity measurements. X-ray reflectivity shows that all films are covered with an oxide layer of about $20 \AA$ in ambient atmosphere. The (110) texture at high $T_{\mathrm{S}}$ decreases towards lower $T_{\mathrm{S}}$, accompanied by an increasing surface roughness. Below $T_{\mathrm{S}}=350^{\circ} \mathrm{C}$ the grain size in the growth direction becomes smaller than the film thickness and a relaxation of intrinsic stress is observed. A transition from a columnar growth structure to a fine grained microstructure is inferred. The change of the microstructure with $T_{\mathrm{S}}$ is probably due to the temperature dependent grain boundary mobility during the deposition process.
\end{abstract}

\section{Introduction}

The preparation of thin niobium films on sapphire is a subject of continuing interest for the study of superconductivity and epitaxial growth $[1-7]$. The electrical transport porperties and the superconducting transition temperature $T_{\mathrm{c}}$ depend strongly on substrate orientation and film thickness $D$. An influence of $T_{\mathrm{S}}$ on the resistivity of $\mathrm{Nb}$ films has been reported for $T_{\mathrm{S}}=450$ $750^{\circ} \mathrm{C}[6]$. For thin $\mathrm{Nb}$ films $(D<40 \AA)$ on sapphire (1T02), strained-layer epitaxy was observed when the substrate was cleaned by ion bombardment prior to the deposition [7].

The crucial influence of $T_{\mathrm{S}}$ on the microstructure of thin metallic films has been observed for many film/substrate combinations [8-10]. An empirical scheme was proposed, in which the type of grain growth and microstructure in vapour-deposited films is classified in different zones depending on the reduced temperature $T_{\mathrm{S}} / T_{\mathrm{M}}\left(T_{\mathrm{M}}\right.$, melting temperature) [8-10]. This structure-zone model has been supported by computer simulations $[11,12]$ and has become a general classification scheme for the study of many film properties including hardness, corrosion and electrical conductivity.

In order to elucidate the influence of $T_{\mathrm{S}}$ on the growth morphology of $\mathrm{Nb}$, we investigated in detail the structure of vapour-deposited films on sapphire prepared at different $T_{\mathrm{S}}$ values and constant film thickness. Our results are complemented by measurements of the electrical resistivity and superconducting transition temperature as functions of $T_{\mathrm{S}}$.

\section{Experimental details}

Films of thickness $250 \AA$ were grown on sapphire (1120) in an ultra-high vacuum system by electronbeam evaporation of high purity niobium (99.99\%, Metallwerk Plansee, Reutte, Austria). The details of the system have been described elsewhere [13]. Prior to the deposition process the substrate was heated, sputter etched and annealed to obtain a clean single-crystalline surface as checked by Auger analysis. The molybdenum sample holder could be heated to substrate temperatures up to $750^{\circ} \mathrm{C}$ controlled by a $\mathrm{W} / \mathrm{Re}$ thermocouple. Thickness and deposition rate (typically $1 \AA^{-1}$ ) were controlled with a quartz crystal monitor.

The epitaxial relationship between sapphire and niobium has been reported by several authors [3-6]. On sapphire (1120), $\mathrm{Nb}$ grows parallel to the (110) lattice planes with an in-plane orientation of the two orthogonal axes $\mathrm{Nb}$ [111] $\| \mathrm{Al}_{2} \mathrm{O}_{3}[0001]$ and $\mathrm{Nb}[112] \| \mathrm{Al}_{2} \mathrm{O}_{3}$ [1100]. The misfit strain between sapphire and $\mathrm{Nb}$, i.e. $1 \%$ and $2 \%$ tensile strain in the growth plane along the sapphire [0001] and [1100] directions, is relaxed in films with $D$ larger than the critical thickness (about $100 \AA$ ) for pseudomorphic growth by the formation of misfit dislocations [14].

Structural characterization was done ex situ by means of X-ray diffraction in a conventional powder diffractometer (Siemens D 500 ). $\mathrm{CuK} x$ radiation $(\lambda=1.54 \AA)$ was collimated with slits of $0.1^{\circ}$ and $0.018^{\circ}$ width in the incident and diffracted beams respectively. The graphite secondary monochromator reduced only 
the background and $\mathrm{K}_{\beta}$ radiation, not the contribution of the $K \alpha_{2}$ line. The resolution of the diffractometer was $\Delta 2 \Theta=0.03^{\circ}$ at $2 \Theta=37.8^{\circ}$ as obtained from the resolved $\mathrm{K} \alpha_{1}$ line width of the sapphire (1120) reflection.

Longitudinal scans (Bragg scans) of the $\mathrm{Nb}$ (110) reflection with the scattering vector parallel to the growth direction where performed in $\Theta / 2 \Theta$ mode. For measuring the rocking curves (transverse scans, where the scattering vector of constant absolute value runs almost parallel to the surface) the scintillation counter was set fixed at the Bragg position $\Theta_{B}$ and the sample was rotated in $\Theta$ with the rocking angle $\omega=2 \Theta-2 \Theta_{\mathrm{B}}$.

The electrical resistivity and superconducting transition temperature were measured with an a.c. bridge and four-point probe method. The temperature was controlled with a calibrated carbon-glass thermometer with an accuracy of $10 \mathrm{mK}$ at $10 \mathrm{~K}$.

\section{Results}

\subsection{Bragg scattering}

Figure 1 shows a selection of representative scans for the (110) reflection with a logarithmic intensity scale for different substrate temperatures $T_{\mathrm{s}}$. The low- $\Theta$ side of the Bragg peak is not shown because of the vicinity of the strong substrate reflection. The high- $\Theta$ side exhibits the so-called Laue oscillations, which are most pronounced for the highest $T_{\mathrm{S}}\left(750^{\circ} \mathrm{C}\right)$. These oscillations arise from the finite number of coherently scattering planes and have also been found in other epitaxial films $[15,16]$. For thin single-crystalline films where the

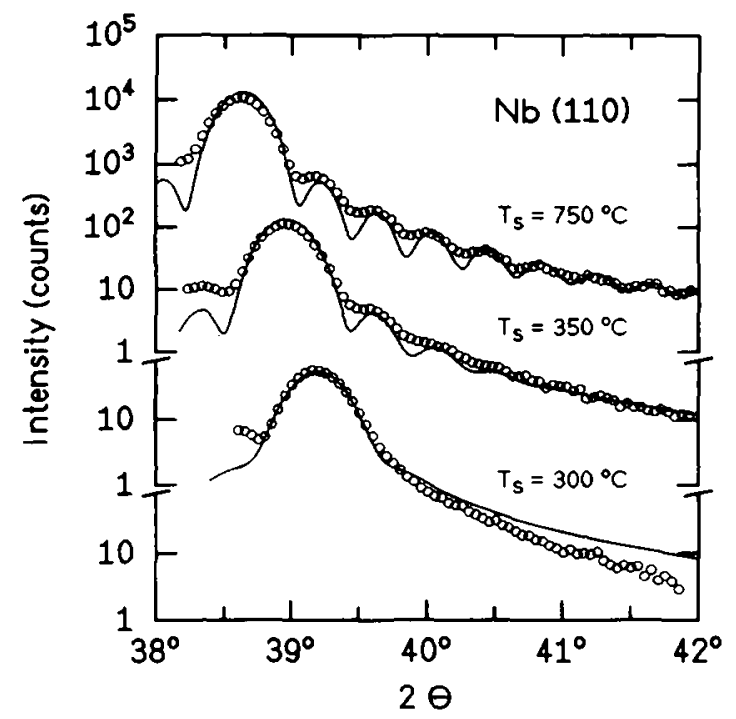

Fig. 1. X-ray diffraction scans of $250 \AA \mathrm{Nb}$ films prepared at different substrate temperatures $T_{\mathrm{s}}$. The intensity is plotted on a log scale. The solid line is a calculation of the line profile after eqn. (1). coherent scattering distance (i.e. grain size) in the growth direction is equal to the film thickness, the period of the Laue oscillations allows a direct determination of the film thickness. A fluctuation of the coherent scattering distance due to surface and/or interface roughness leads to a damping of the oscillation amplitude with increasing distance from the fundamental Bragg reflection. Figure 1 clearly shows that the roughness increases with decreasing $T_{\mathrm{S}}$, as expected from the fact that epitaxial films of high quality are usually deposited at enhanced temperatures to promote surface diffusion and thus smooth film growth.

For a more quantitative analysis, the scattered intensity was calculated. Roughness was taken into account by a Gaussian distribution of the number of coherently scattering lattice planes and an incoherent superposition of each Laue function [16].

$$
\begin{aligned}
I(k) \sim & \sum_{N}(1 / \sigma) \exp \left[-\left(N-N_{0}\right)^{2} / \sigma^{2}\right] \\
& \times \sin ^{2}(k N d / 2) / \sin ^{2}(k d / 2)
\end{aligned}
$$

where $k=4 \pi \sin \Theta / \lambda$ is the absolute value of the scattering vector, $N_{0}$ is the average number of scattering planes with a distance $d$, and $\sigma$ is the width of the distribution. Peak broadening due to the $\mathrm{K} \alpha$ doublet was introduced by convoluting eqn. (1) with the instrumental function (resolved $K \alpha$ doublet of the substrate reflection). The solid lines in Fig. 1 represent the calculated intensity with appropriate parameters $d, \sigma$ and $N_{0}$. These parameters can be obtained with some reliability because each one has a distinctive influence on the diffraction pattern: $d$ corresponds to the angular position of the Bragg reflection and $N_{0}$ and $\sigma$ determine the period and damping respectively of the oscillations. The calculations describe the maxima of the Bragg peak and Laue oscillations quite well, although the sharp minima are not fully reproduced experimentally, possibly because of the presence of inhomogeneous strains. This should be checked with high-resolution X-ray diffraction. Very good agreement with the theoretical pattern has been obtained for Co films on sapphire using high-resolution X-ray diffraction [16]. The observed behaviour is not altered when the diffuse background scattering is subtracted. A sudden wash-out of the oscillations is clearly seen on going from $350^{\circ} \mathrm{C}$ to $300^{\circ} \mathrm{C}$.

The lattice parameter $d$, the average size of coherent scattering grains in the growth direction $N_{0} d$, and the roughness parameter $\sigma$ are plotted vs. $T_{\mathrm{S}}$ in Fig. 2. At $750{ }^{\circ} \mathrm{C}, d$ is nearly equal to the bulk value $(2.3325 \AA)$. For lower $T_{\mathrm{s}}, d$ decreases towards a minimum at $T_{\mathrm{S}}=300^{\circ} \mathrm{C}$, corresponding to a maximum compression of $\varepsilon \approx-1.5 \%$ in the growth direction. A steep increase of $d$ to the bulk value occurs with further decreasing $T_{\mathrm{s}}$. The effect of different thermal expansion coefficients 


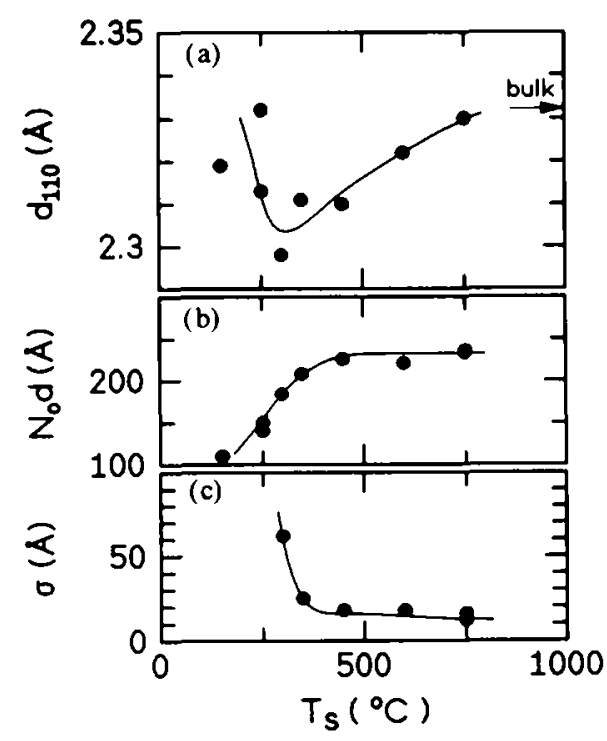

Fig. 2. (a) Lattice constant $d_{110}$, (b) grain size $N_{0} d$, (c) width of the grain size distribution $\sigma$ as obtained from the profile calculation (eqn. 1) vs. substrate temperature $T_{\mathrm{s}}$.

between substrate and film [17] yields a very small tensile strain which cannot explain this behaviour. The variation must be attributed to a different generation and relaxation of growth stress (intrinsic stress) at low and high $T_{\mathrm{s}}$. Epitaxial strains can be neglected for $D>100 \AA[14]$ as mentioned above.

At high $T_{\mathrm{S}}, N_{0} d$ is almost equal to the film thickness (Fig. 2(b)), representing the growth of epitaxial grains connecting the film boundaries with an average roughness of the substrate-film and film-air interfaces of about $10 \AA$, which increases with decreasing $T_{\mathrm{S}}$ (Fig. $2(c))$. When the grain size falls below the film thickness $\left(T_{\mathrm{S}}<450^{\circ} \mathrm{C}\right)$, there is more than one grain connecting the two film boundaries and $\sigma$ is a measure of the variation of grain sizes in the film rather than a measure of surface roughness. This is suggested by the steep increase of $\sigma$ towards the lowest $T_{\mathrm{s}}$.

Figure 3 shows the rocking curve of the $\mathrm{Nb}$ (110) reflection for $T_{\mathrm{S}}=750^{\circ} \mathrm{C}$, which exhibits a two-component line shape. The narrow component with a full width at half maximum (FWHM) $\Delta \omega_{1}=0.03^{\circ}$ is resolution limited. The broad background has an FWHM of $\Delta \omega \approx 0.5^{\circ}$. This kind of profile has been observed for all films with, however, a change of the intensity ratio $P$ between the integrated intensities of the narrow and broad components. Neglecting a contribution from the orientational distribution of epitaxial crystallites (mosaicity), the width of the rocking curve gives the lower limit for the lateral domain size $L=\lambda / 2 \Delta \omega \sin \Theta$ [17]. We obtain $L_{1} \geqslant 4500 \AA$ from the narrow and $L_{2} \geqslant 270 \AA$ from the broad component.

The occurrence of a two-component rocking curve has also been reported by Reimer et al. for $\mathrm{Nb}$ films

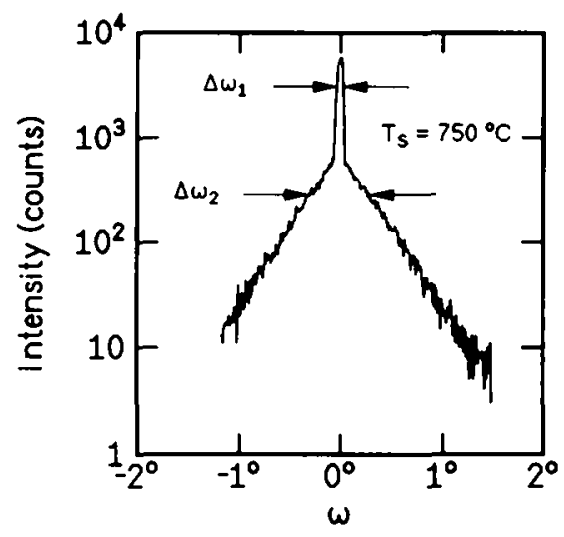

Fig. 3. Rocking curve of a $250 \AA \mathrm{Nb}$ film deposited at $T_{\mathrm{S}}=750^{\circ} \mathrm{C}$. The intensity is plotted on a log scale.

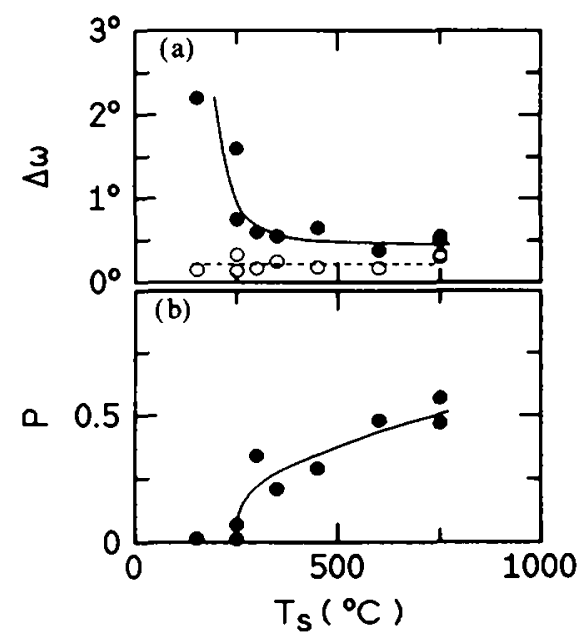

Fig. 4. (a) Widths (FWHM) of the narrow and broad component of the rocking curves: $\bigcirc, \Delta \omega_{1} \times 5 ;-\Delta \omega_{2} ;$ (b) intensity ratio $P$ between the integrated intensities of the narrow and broad component.

950-3000 $\AA$ and $T_{\mathrm{S}}=850^{\circ} \mathrm{C}$, using high resolution $\mathrm{X}$ ray diffraction [18]. They suggested that the narrow component is due to grains with their lattice planes strongly coupled to the substrate, whereas the broad component represents the "bulklike" portion of the film, i.e. independent grains not coupled to the substrate. Both contributions seemed to originate from the same depth region with the same vertical coherence length.

The effect of $T_{\mathrm{S}}$ on $P$ and the line widths $\Delta \omega_{1}$ and $\Delta \omega_{2}$ is shown in Fig. 4. $\Delta \omega_{1}$ is independent of the film thickness for all $T_{\mathrm{s}}$. The increase in $\Delta \omega_{2}$ and the accompanying decrease in $P$ when the film is deposited at lower $T_{\mathrm{s}}$ can be interpreted as a variation of grain size and texture, with the tendency to prevent the growth of epitaxial grains strongly coupled to the substrate at the expense of the bulklike portion. The strong variation of all parameters at about $T_{\mathrm{S}}=300^{\circ} \mathrm{C}$, corre- 


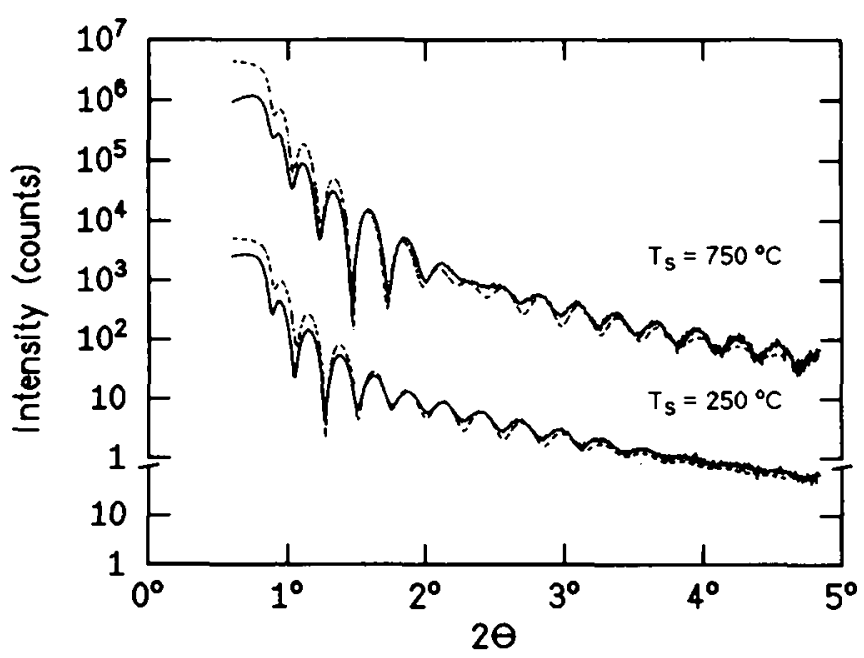

Fig. 5. Small angle $\mathrm{X}$-ray reflectivity for two $\mathrm{Nb}$ films prepared at high and low $T_{\mathrm{s}}$. The intensity is plotted on a log scale. The broken lines show the calculated reflectivity.

sponding to $T_{\mathrm{S}} / T_{\mathrm{M}} \approx 0.22$ (melting temperature $T_{\mathrm{M}}=2741 \mathrm{~K}$ ) seems to be due to a change in the microstructure of the films.

\subsection{Small angle scattering}

$\mathrm{X}$-ray reflectivity measurements at small angles provide information about the electron density profile normal to the film independent of the crystalline structure. Figure 5 shows the reflected intensity in a logarithmic scale for scattering angles larger than the critical angle of total external reflection $\left(\Theta_{c}(\mathrm{Nb})=0.39^{\circ}\right)$. The diagram was obtained by joining several patterns with different beam power to ensure that the error introduced by the dead time of the scintillation counter $(1 \mu \mathrm{s})$ is small. The data do not change much when the diffuse background is substracted. Several interference maxima are visible. An oxide layer, which unavoidably builds up on the film surface in ambient atmosphere, yields a kind of "beating" which is best observed for $T_{\mathrm{S}}=750^{\circ} \mathrm{C}$. Again, film roughness causes a damping of the oscillations with increasing angle as compared with the ideal case of a perfectly flat surface.

In order to estimate the thickness of the oxide layer and the amplitude of roughness, the data were compared to a theoretical fit. The reflectivity of a smooth film covered with an oxide layer on a substrate of infinite thickness was calculated with a recursion formula of Parratt [19]. The oxidation of $\mathrm{Nb}$ at room temperature has been studied in detail by Halbritter $[20,21]$. In general $\mathrm{Nb}$ is coated with about one monolayer $\mathrm{NbO}$ and up to $20 \AA \mathrm{Nb}_{2} \mathrm{O}_{5}$. The nucleation of $\mathrm{Nb}_{2} \mathrm{O}_{5}$ crystallites introduces large strains into the interface because of a volume increase by more than a factor of 3. This strain is relaxed by $\mathrm{NbO}_{6}$ precipitations at the interface and by a serration of the $\mathrm{Nb}$ surface.
Therefore we assumed the formation of $\mathrm{Nb}_{2} \mathrm{O}_{5}$ for the calculation of the reflectivity. Roughness was taken into account after the model of Névot and Croce [22], yielding a modified Fresnel reflectivity for each interface. The calculation can be extended for the case of metallic multilayers [23]. Figure 5 shows a comparison between the measurement and the model. From the calculated intensity we obtain an oxide layer thickness of 19-24 $\AA$ for all samples. This is in good agreement with the superconducting and XPS measurements [20, 21].

For $T_{\mathrm{S}}=750{ }^{\circ} \mathrm{C}$ the $\mathrm{Nb}$ layer thickness agrees within $3 \%$ with the crystallite size as estimated from the Laue oscillations. The surprising result is that the roughness parameters do not depend crucially on $T_{\mathrm{S}}$. Best fits were obtained with $\sigma_{\text {sub }}=1 \AA, \sigma_{\text {ox }}=9 \AA$ and $\sigma_{\mathrm{Nb}}=9 \AA$ $\left(T_{\mathrm{S}}=750^{\circ} \mathrm{C}\right)$ or $13 \AA\left(250^{\circ} \mathrm{C}\right)$. For $T_{\mathrm{S}}=750^{\circ} \mathrm{C}$ the overall roughness is nearly equal to $\sigma$ obtained from the Bragg scattering. The small roughness at lower $T_{\mathrm{S}}$ $\left(250^{\circ} \mathrm{C}\right)$ seems contradictory to what is expected from the limited adatom mobility at low $T_{\mathrm{s}}$. A simple explanation is that the two methods, i.e. reflectivity and Bragg scattering, measure different properties. The Laue oscillations give an estimate of the variation of grain sizes in the growth direction, whereas the reflectivity is sensitive only to the roughness amplitude of the two film boundaries. In addition it should be mentioned that the model of Névot and Croce is applicable only to Gaussian height-height correlations. This simple assumption seems not to be valid for the oxidized $\mathrm{Nb}$ surface since the serration yields a more complicated surface profile.

\subsection{Electrical resistivity}

Figure 6 shows the residual electrical resistivity $\rho_{10}$ at $T=10 \mathrm{~K} v$ s. substrate temperature $T_{\mathrm{s}}$. Our data extrap-

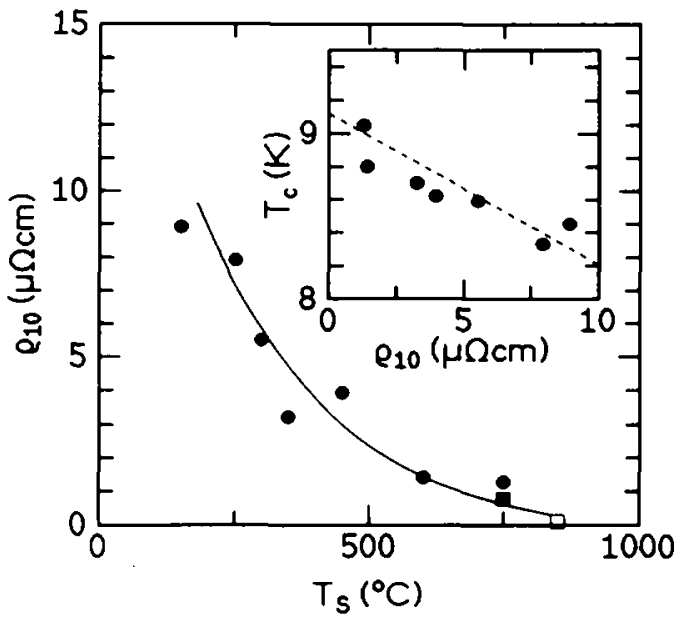

Fig. 6. Residual resistivity $\rho_{10}$ at $T=10 \mathrm{~K}$ vs. $T_{\mathrm{S}}$ : (O) this work; (更) $D=2600 \AA[24] ;(\ulcorner) d=6000 \AA$ [4]. The inset shows the dependence of the superconducting transition temperature $T_{\mathrm{c}}$ on $\rho_{10}$. The broken line shows the universal behaviour for $1500 \AA \mathrm{Nb}$ films after ref. 27. 
trapolate well to the data of $\mathrm{Nb}$ films with higher $T_{\mathrm{S}}=850^{\circ} \mathrm{C}$ and $D=100-6000 \AA[4,5]$.

In general, the residual resitivity of thin films is influenced by scattering of conduction electrons from several kinds of defect such as point defects, dislocations, grain boundaries, and the film surfaces [25]. Scattering at the film surfaces is usually interpreted in terms of the Fuchs-Sondheimer theory, leading to a thickness dependent contribution $\rho(D)$. In addition, grain-boundary scattering has to be taken into account in most cases [26]. Although there may be other contributions to the resistivity that depend on $T_{\mathrm{S}}$, such as the shape and orientation of the crystallites, the overall behavior of $\rho_{10}$ with $T_{\mathrm{S}}$ can easily be explained by a decreasing grain size with decreasing temperature in agreement with the $\mathrm{X}$-ray diffraction analysis.

The dependence of the resistivity on $T_{\mathrm{S}}$ could also be due to different amounts of $\mathrm{Nb}$ oxides when the samples are exposed in air. It has been shown that the serration by oxidation is enhanced when defects in the upper $\mathrm{Nb}_{2} \mathrm{O}_{5}$ layer come into play. These defects are nucleated by defects of the initially unexposed $\mathrm{Nb}$ surface $[20,21]$. Therefore, in any case the origin of the dependence of $\rho_{10}\left(T_{\mathrm{S}}\right)$ is an increasing defect density of the original $\mathrm{Nb}$ film with decreasing $T_{\mathrm{S}}$.

The superconducting transition temperature $T_{\mathrm{c}}$ is directly related to $\rho_{10}$ (see inset of Fig. 6). Our data are close to the "universal plot" $T_{\mathrm{c}}\left(\rho_{10}\right)$ reported by Park et al. [27] (broken line), which was obtained for $1500 \AA$ $\mathrm{Nb}$ films, each prepared in a different manner under various evaporation conditions in order to vary the structural quality. This line describes also the data of $\mathrm{N}^{-}$-ion implanted $\mathrm{Nb}$ films [28]. For $\rho_{10} \ll 100 \mu \Omega \mathrm{cm}$ the depression of $T_{\mathrm{c}}$ has been attributed to the broadening of the single-particle density of states at the Fermi level with increasing disorder. The good agreement between the data shows nicely the influence of disorder introduced by the growth process. Concerning the oxide layer, it has been shown that $T_{\mathrm{c}}$ was lowered only for $D<100 \AA$. Localization effects are found to be important for much larger residual resistivities above $100 \mu \Omega \mathrm{cm}[28]$.

\section{Discussion}

The strong variation of the structural data with $T_{\mathrm{S}}$ implies a change in the microstructure of the $\mathrm{Nb}$ films depending on substrate temperature. There has been a lot of experimental work on the grain structure of vapour-deposited metallic films. For thick deposits and high deposition rates $\left(R>100 \AA \mathrm{s}^{-1}\right)$, three temperature zones have been found, each of which is characterized by a different type of microstructure $[9,10]$. For $T_{\mathrm{S}}<0.3 T_{\mathrm{M}}$ (zone I), film growth proceeds by ballistic aggregation and the internal structure is porous and contains a high dislocation density. For $0.3<T_{\mathrm{S}}<0.5$ (zone II), columnar grains which extend vertically through the film thickness are formed. The lateral grain size increases with increasing $T_{\mathrm{s}}$ and the microstructure is mainly controlled by a large surface diffusion. This columnar structure is most clearly seen for oblique incidence of the vapour beam, but has also been found for normal incidence [29]. For higher $T_{\mathrm{S}}>0.5 T_{\mathrm{M}}$ (zone III), the film consists of large grains with a diameter exceeding the film thickness.

Although this classification scheme has been applied for high deposition rates, a computer simulation shows that the transition temperature $T_{1}$ between zones I and II depends on the deposition rate [11]. The slower surface diffusion at lower $T_{\mathrm{S}}$ can be compensated by a larger time for the migration of adatoms to build up a monolayer. From the experimentally established boundary temperature $T_{1} \approx 0.3 T_{\mathrm{M}}$ for $R \approx 1000 \AA \mathrm{s}^{-1}$, a smaller $T_{1} \approx 0.21 T_{\mathrm{M}}$ is obtained for $R=1 \AA \mathrm{s}^{-1}$ [11]. The almost perfect agreement with the transition at $T_{\mathrm{S}} \approx 0.22 T_{\mathrm{M}}$ for the Nb films should not be taken too seriously, but shows that the zone model should also be applicable for lower deposition rates.

A further study on the grain structure of several evaporated films proposes a refinement of the zone model and takes into account a gradual transition between I and II with a bimodal grain size distribution of large grains surrounded by grains of the same size found at lower $T_{\mathrm{s}}$ [8]. The variation of grain structure with $T_{\mathrm{S}}$ was explained by a dual process of grain growth and granular epitaxy during the deposition process. The important variable is the mobility of the individual grain boundaries, which is influenced by the substrate temperature. This model is able to explain the observed grain structures in many metallic films [8].

In comparison with the results of the $\mathrm{Nb}$ films, we conclude that a columnar grain structure with a smooth surface builds up for high temperatures with a grain size in the growth direction equal to the film thickness. From the resistivity data we suppose that the lateral grain size in this regime is larger than $D$. By lowering $T_{\mathrm{S}}$ one enters the transition zone around $T_{\mathrm{S}}=300^{\circ} \mathrm{C}$, in which the grain structure contains a bimodal distribution of columns and fine equiaxed grains and the average grain size $N_{0} d$ decreases steeply. The aligned growth in the (110) direction persists at low temperatures because the texture is determined by the early stages of growth on the substrate and only the angular distribution changes.

The observed compression of the lattice parameter for $300{ }^{\circ} \mathrm{C}<T_{\mathrm{S}}<750{ }^{\circ} \mathrm{C}$ corresponds to a tensile strain in the film plane due to the Poisson's ratio. Intrinsic stresses have been also observed for other metallic films 
[30]. In $\mathrm{Nb}$ films a specific relaxation process seems to occur around $300^{\circ} \mathrm{C}$. Such a relaxation mechanism could be associated with the change of the grain structure. In the columnar regime only the film boundaries serve as sinks and sources for vacancies [31]. The generated stress increases with decreasing temperature due to the decreasing atom mobility. For $T_{\mathrm{S}}<300^{\circ} \mathrm{C}$ the presence of grain boundaries parallel to the film surface and the refinement of the grain structure probably allows a stress relaxation via grain boundary diffusion [31]. However, a detailed investigation of the stress behaviour must be conducted to confirm this suggestion.

In conclusion, we found a correlated variation of several structural parameters with substrate temperature $T_{\mathrm{S}}$. This is explained by a change of the microstructure due to the effect of $T_{\mathrm{S}}$ on the grain growth and grain boundary mobility. The behaviour seems to be universal for many metallic films when compared with the reduced temperature $T_{\mathrm{S}} / T_{\mathrm{M}}$ [8]. The disorder introduced by the growth process at low $T_{\mathrm{S}}$ also influences the resistivity and superconducting transition temperature $T_{\mathrm{c}}$.

\section{Acknowledgments}

We would like to thank J. Halbritter, R. ManailaDevenyi and J. Woitok for helpful discussions. We also thank E. Bieringer and T. Trappmann for their assistance during the sample preparation. This work was supported by the Deutsche Forschungsgemeinschaft.

\section{References}

1 S. I. Park and T. H. Geballe, Phys. Rev. Lett., 57 (1986) 901

2 J. W. P. Hsu and A. Kapitulnik, Phys. Rev., B45 (1992) 4819

3 S. M. Durbin, J. E. Cunningham, M. E. Mochel and C. P. Flynn, J. Phys., Fll (1981) L223.
4 S. A. Wolf, S. B. Qadri, J. H. Claassen, T. L. Francavilla and B. J. Dalrymple, J. Vac. Sci. Technol., A4 (1986) 524.

5 J. H. Claassen, S. B. Qadri, P. R. Broussard and S. A. Wolf, in M. Hong, S. A. Wolf and D. C. Gubser (eds.), Metallic Multilayers and Epitaxy, The Metal Society, Warendale, PA, 1988, p. 217.

6 G. I. Oya, M. Koishi and Y. Sawada, J. Appl. Phys., 60(1986) 1440.

7 S. I. Park, A. Marshall, R. H. Hammond, T. H. Geballe and J. Talvacchio, J. Mater. Res., 2 (1987) 446.

8 C. R. M. Grovenor, H. T. G. Hentzell and D. A. Smith, Acta Metall., 32 (1984) 773.

9 B. A. Movchan and A. V. Demchishin, Phys. Met. Metallogr., 28 (1969) 83

10 J. A. Thornton, Ann Rev. Mat. Sci., 7 (1977) 239.

11 K. H. Müller, J. Appl. Phys., 58 (1985) 2573.

12 A. Mazor, D. J. Srolovitz, P. S. Hagen and B. G. Bukiet, Phys. Rev. Lett., 60 (1988) 424.

13 C. Sürgers and H. v. Löhneysen. Thin Solid Films, 219 (1992) 69.

14 F. H. Lamelas, H. He and R. Clarke, Phys. Rev., B38 (1988) 6334.

15 P. F. Micheli, C. J. Palmström and K. W. Moyers, Appl. Phys. Lett., 58 (1991) 1602

16 A. Stierle, A. Abromeit, K. Bröhl, N. Metoki and H. Zabel, in H. Zabel and I. K. Robinson (eds.), Surface $X$-ray and Neutron Scattering, Proceedings in Physics, Vol. 61, Springer Verlag, Berlin, 1992, p. 203.

17 A. Segmüller, I. C. Noyan and V. S. Speriosu, Prog. Cryst. Growth Charact., 18 (1989) 21.

18 P. M. Reimer, H. Zabel, C. P. Flynn and J. A. Dura, Phys. Rev., B45 (1992) 11426

19 L. G. Parratt, Phys. Rev., 95 (1954) 359.

20 J. Halbritter, Appl. Phys., A43 (1987) 1.

21 J. Halbritter, J. Less Common Met., 139 (1988) 133.

22 L. Névot and P. Croce, Rev. Phys. Appl,, 15 (1980) 761.

23 R. Manaila-Devenyi, C. Sürgers, H. Menges and H. v. Löhneysen, Rev. Roum. Phys., 36 (1991) 735.

24 C. Strunk, Ph.D. Thesis, Universität Karlsruhe, 1992.

25 D. Schumacher. Surface Scattering with Conduction Electrons, Tracts in Modern Physics, Vol. 128, Springer Verlag, Berlin, 1993.

26 A. F. Mayadas and M. Shatzkes, Phys. Rev., BI (1970) 1382.

27 S. I. Park and T. H. Geballe, Physica, B135 (1985) 108.

28 C. Camerlingo, P. Scardi, C. Tosello and R. Vaglio, Phys. Rev. B3I (1985) 3121

29 A. G. Dirks and H. J. Leamy, Thin Solid Films, 47 (1977) 219 and references therin.

30 E. Klokholm, J. Vac. Sci. Technol., 6 (1969) 138.

31 M. F. Doerner and W. D. Nix, CRC Crit. Rev. Solid State Mater. Sci., 14 (1988) 225. 